\title{
A Rare Case of a Giant Congenital Multinodular Goiter with Intrathyroidal Parathyroid Adenoma
}

\author{
R Lakshmi Menon, ${ }^{1}$ M Muniraju ${ }^{2}$
}

\begin{abstract}
Aim: To highlight the management of huge multinodular goiters and report a rare case of intrathyroidal parathyroid adenoma.

Background: Goiter is an abnormal enlargement of thyroid gland. Huge goiters are a surgical challenge due to possible difficulty in intubation, tracheomalacia, retrosternal extension and distorted anatomy. Goiters associated with congenital hypothyroidism undetected at birth leads to permanent neurological deficits. This case report highlights a case of huge multinodular goiter with a rare intrathyroidal parathyroid adenoma detected on histopathology.

Case description: This case report deals with a 27-year-old male patient, presenting with huge hypothyroid multinodular goiter weighing $750 \mathrm{~g}$ with no compression symptoms. He had low IQ probably due to undetected hypothyroidism at birth. His postoperative histopathology report showed an intrathyroidal parathyroid adenoma which is an extremely rare tumor.

Conclusion: This report aims to emphasize the existence of congenital hypothyroidism and also that huge goiters when dissected meticulously may be removed without any major complications. We also report an incidental finding of rare intrathyroidal parathyroid adenoma.

Clinical significance: Neonatal screening of hypothyroidism and its subsequent treatment can prevent mental retardation. We would also like to emphasize the importance of preoperative serum parathormone and calcium levels in all patients undergoing thyroidectomy to detect asymptomatic parathyroid adenomas.

Keywords: Congenital goiter, Huge goiter, Intrathyroid adenoma, Parathyroid adenoma, Thyroid.

International Journal of Head and Neck Surgery (2020): 10.5005/jp-journals-10001-1387
\end{abstract}

\section{BACKGROUND}

Goiter is an abnormal enlargement of thyroid gland. ${ }^{1}$ A benign nodular goiter usually does not cause obstructive symptoms like involvement of trachea, esophagus or recurrent laryngeal nerve. ${ }^{2}$

Congenital hypothyroidism is the most common congenital endocrine disorder commonly attributable to iodine deficiency in mothers. ${ }^{3}$ Endemic cretinism is more common in areas of Southern Europe, Bangladesh, China, India and Nepal. ${ }^{4}$ It is characterized by severely stunted physical and mental growth. It may be of two types; neurological and myxedematous. Morbidity may be reduced by early diagnosis and supplementation with thyroxine. ${ }^{4}$

Giant goiters weighing more than $500 \mathrm{~g}$ are associated with surgical challenge due to possible tracheomalacia, retrosternal extension, difficulty in intubation and intraoperative dissection of thyroid gland. ${ }^{7}$

Parathyroid adenomas are classified into adenomas, hyperplasia and carcinoma. Hyperparathyroidism presents with bone fracture, calculi of urinary tract and high serum calcium levels. ${ }^{5}$ Ultrasonography and radionucleotide (Tc99-sestamibi) imaging are tools of choice in diagnosing parathyroid adenoma. ${ }^{6}$ The prevalence of intrathyroidal parathyroid adenoma varies from $1.4 \%$ to $6 \%$ which results from abnormality during migration of parathyroid resulting in entrapment within thyroid gland. The use of SPECT provides excellent information on ectopic ademona. ${ }^{6}$

In this case report, we intend to discuss a case of huge multinodular goiter with history of cretinism and postoperative histopathology showing features of intrathyroidal parathyroid adenoma.

\section{Case Description}

A 27-year-old male patient presented to ENT OPD of our hospital with complaints of swelling in front of the neck since childhood

\footnotetext{
1,2 Department of ENT, Ambedkar Medical College, Bengaluru, Karnataka, India
}

Corresponding Author: R Lakshmi Menon, Department of ENT, Ambedkar Medical College, Bengaluru, Karnataka, India, Phone: +91 8123468629, e-mail: drlakshmi51@gmail.com

How to cite this article: Menon RL, Muniraju M. A Rare Case of a Giant Congenital Multinodular Goiter with Intrathyroidal Parathyroid Adenoma. Int J Head Neck Surg 2020;11(1):12-15.

Source of support: Nil

Conflict of interest: None

(Figs 1 and 2). The swelling grew slowly over the years to the present size. There was no history of any pressure symptoms. Due to delayed milestones and macroglossia, he was detected with hypothyroidism at 1 year of age and started on thyroxine supplements. At the time of examination, he was on thyroxine $100 \mu \mathrm{g}$ daily. He gave history of 2 previous failed attempts at intubation for thyroidectomy in the previous hospitals and hence deferred surgery for a while.

General examination revealed a low IQ, pulse rate of 108/ minute, BP-130/80 mm Hg. On examination of the neck, a $15 \times$ $10 \mathrm{~cm}$ irregular swelling was noted extending vertically from at the level of hyoid superiorly to overhanging the clavicle inferiorly, and horizontally between the posterior borders of sternocleidomastoid. The lower border of swelling was palpable and we could get below the swelling. Carotids pulsations were more laterally palpable. No cervical lymphadenopathy noted. Indirect laryngoscopic examination was normal.

All the routine blood investigations were within normal levels. Pure tone audiometry, serum calcium and echo done were within the normal range. Thyroid function tests revealed mildly reduced 


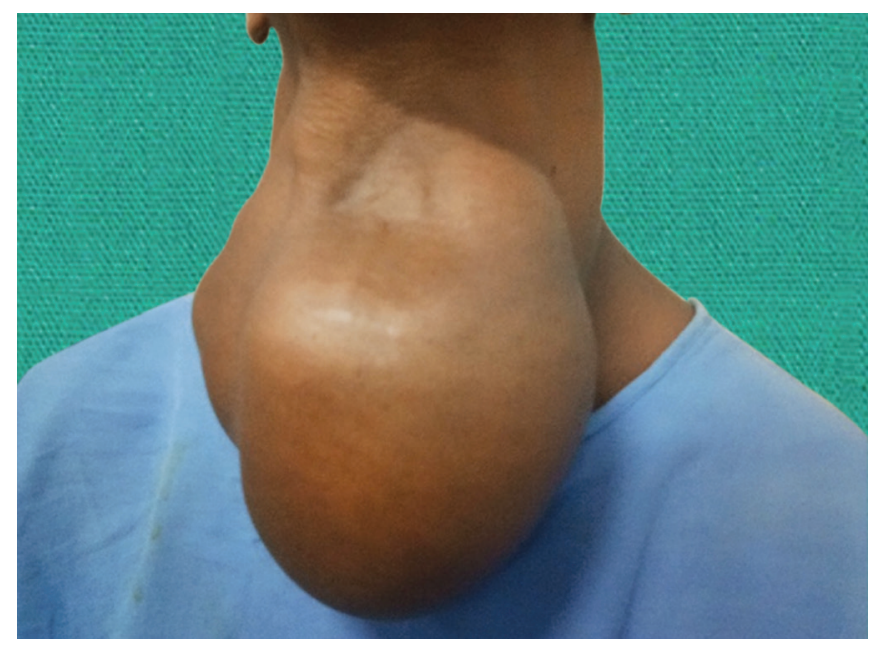

Fig. 1: Huge multinodular goiter (frontal view)

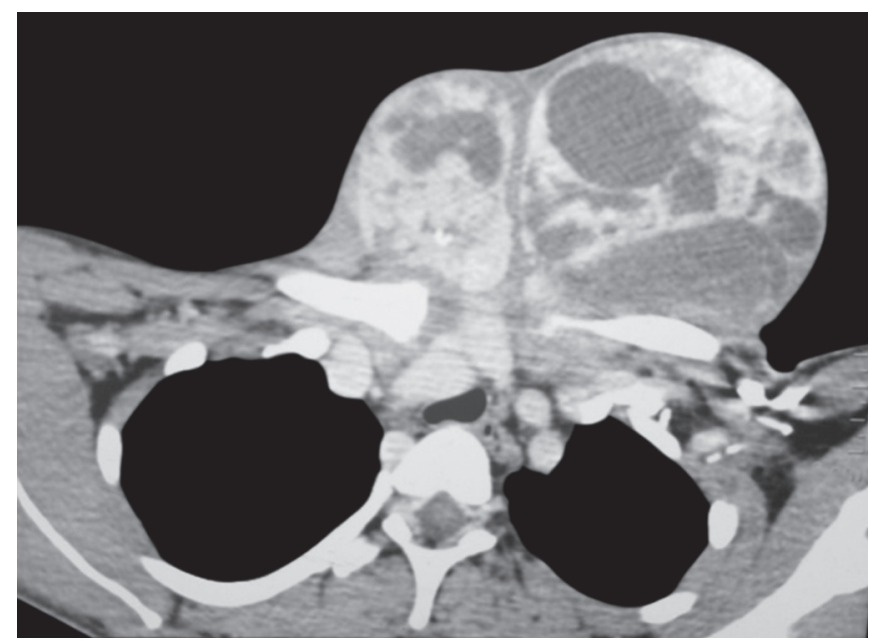

Fig. 3: CT neck (axial) showing multinodular goiter with compression on trachea and displacement of great vessels posteriorly

TSH levels, so thyroxine supplement was reduced to $50 \mu \mathrm{g}$ daily and repeat thyroid function test after a week showed normal results. Fine needle aspiration cytology reported nodular goiter. Computerized tomography of the neck and thorax done revealed a multinodular goiter with laterally displaced great vessels, and a 5 $\mathrm{mm}$ transverse tracheal compression at D5 level with no evidence of malignancy (Figs 3 and 4).

After preanesthetic evaluation, patient was taken up for total thyroidectomy under general anesthesia in lieu of long-standing giant goiter. In view of difficult intubation, patient underwent flexible bronchoscopic guided intubation with flexometallic endotracheal tube sized. ${ }^{7}$

An elliptical skin incision was taken so as to remove the excessive lax skin. The strap muscles were stretched and thinned out and cut for the adequate visualization of the gland. Thyroid lobes were found to be highly vascular with multiple vessels overlying them (Fig. 5). Thyroidectomy was carried on the right and then on left. Due to distorted anatomy, recurrent laryngeal nerve, parathyroid glands could not be identified. The wound was sutured in layers. After inspection of vocal cords, patient was successfully extubated.

The specimen weighed $750 \mathrm{~g}$, with the left lobe weighing $500 \mathrm{~g}$ and right lobe, $250 \mathrm{~g}$ (Fig. 6). The right lobe measured $12 \times 7 \mathrm{~cm}$, and

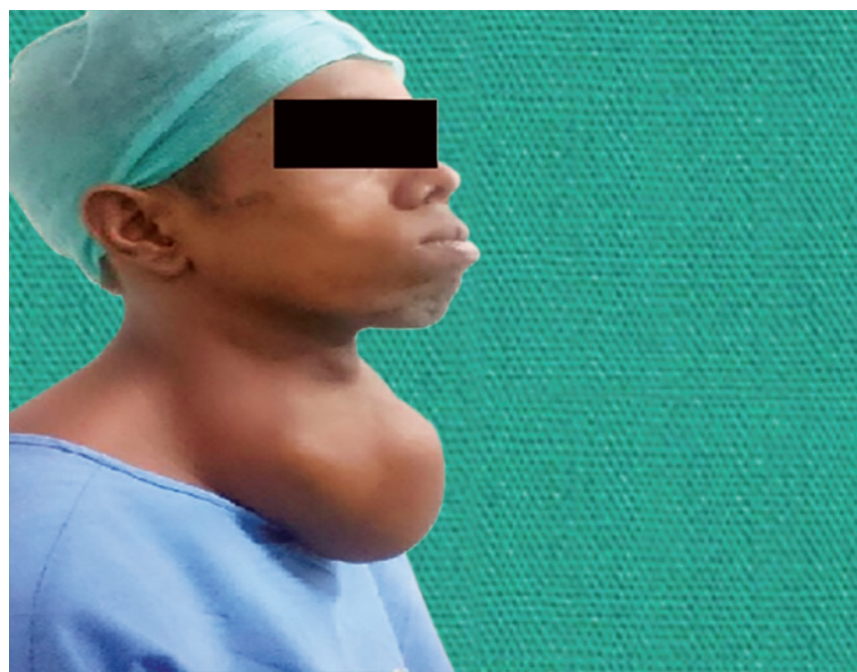

Fig. 2: Huge multinodular goiter (lateral view)

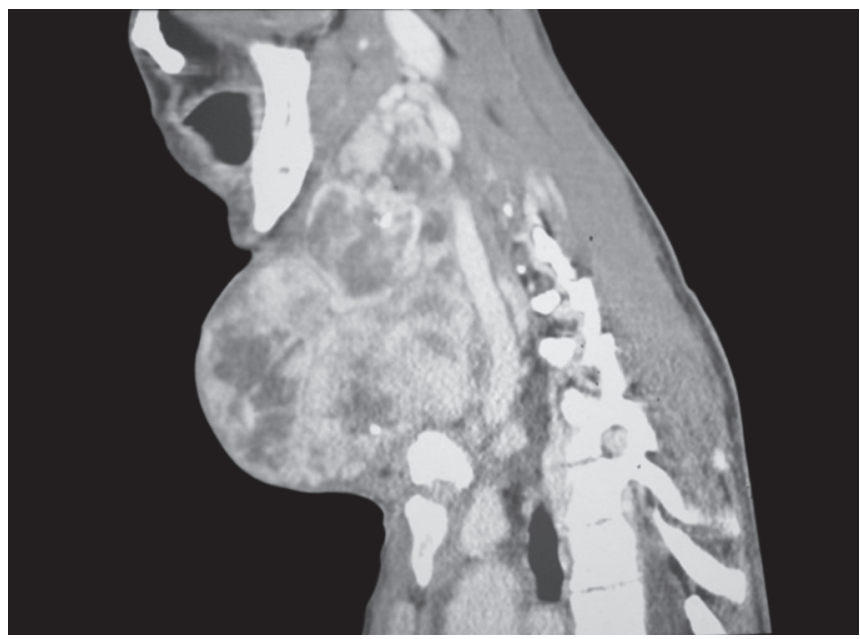

Fig. 4: CT neck (sagittal) demonstrating compression of trachea and great vessels displaced posteriorly

left lobe, $15 \times 10 \mathrm{~cm}$. The specimen was sent for histopathological examination, which revealed multinodular goiter with possibility of intrathyroidal parathyroid adenoma with cystic change (Figs 7 and 8). Since parathyroid adenoma was not suspected prior to surgery, serum parathyroid levels were not assessed.

Postoperative period was uneventful for 72 hours with normal recorded serum calcium. On the fourth postoperative day, patient developed carpopedal spasm. Serum calcium report showed a decrease in serum calcium levels with ionized calcium being $0.90 \mathrm{mmol} / \mathrm{L}$. Hypocalcemia was corrected intravenously with $10 \%$ calcium gluconate. He was also started on oral calcium supplements.

In view of histopathology showing intrathyroidal parathyroid adenoma, a bone dexa scan performed using Tc $99 \mathrm{~m}$ showed an absence of abnormal osteoblastic activity. Ultrasonography of abdomen to rule out MEN syndrome and renal calculi was normal.

\section{Discussion}

Congenital hypothyroidism is reported to be 1 in 3,000-4,000 live births making it the most common congenital endocrine disorder. ${ }^{8}$ Most common reason for congenital hypothyroidism is iodine deficiency in the mother. The intelligence quotient of the 


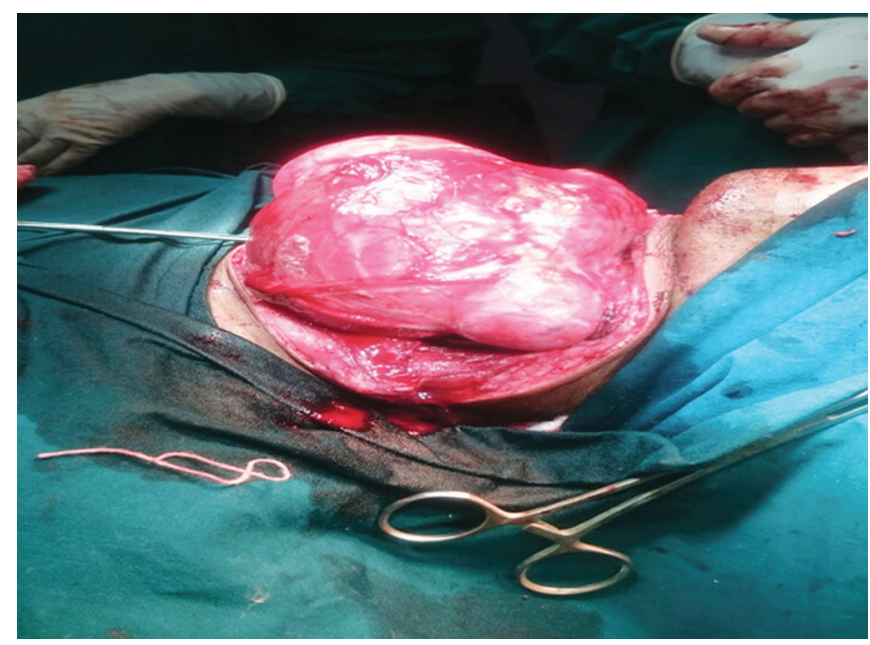

Fig. 5: Intraoperative picture depicting vascularity of thyroid gland

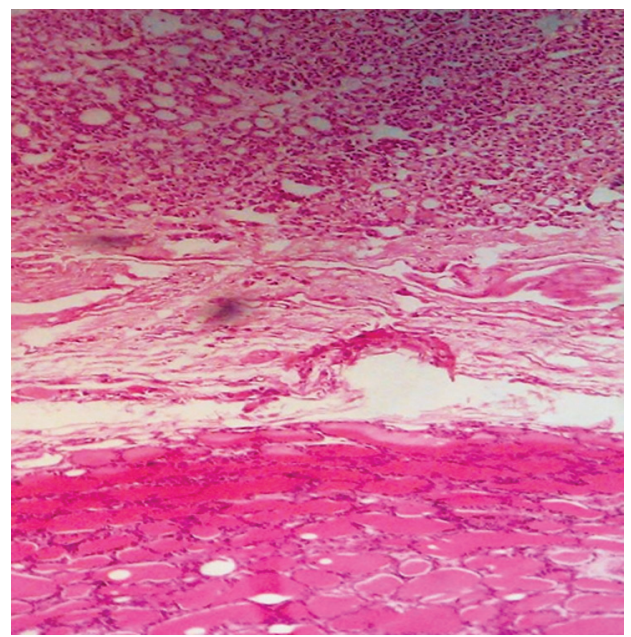

Fig 7: Histopathology showing colloid goiter with parathyroid adenoma

children gets affected depending on the severity of hypothyroidism as determined by thyroid function tests and delayed skeletal maturation at birth. Despite the neonatal screening programmes, $10 \%$ children with severe hypothyroidism needs special education. ${ }^{9}$ In this case report, patient was not screened for hypothyroidism at birth and detected only at 1 year of age due to delayed milestones and macroglossia. Hence the importance of neonatal screening programs to prevent congenital hypothyroidism is vital.

Thyroidectomy for massive goiters is challenging due to multiple risk factors like securing the airway, potential injury to recurrent laryngeal nerve, blood loss, parathyroid injury due to distorted anatomy. ${ }^{7}$ Difficult intubation is anticipated in these patients due to gross tracheal deviation, compression or tracheomalacia. Intubation is achieved using flexometallic endotracheal tubes or tubes of smaller size. ${ }^{10,11}$ Awake intubation with flexible bronchoscopes may be warranted if necessary. ${ }^{12,13}$ In our case, patient could not be intubated twice previously in two other hospitals due to respiratory distress. Hence in our institute, the anesthetists intubated the patient under guidance of flexible bronchoscopy in view of tracheal compression at D5 level.

Primary hyperparathyroidism is a rare disease with hyperparathyroid crisis being an unusual presentation. ${ }^{11,14}$ Increased levels of parathyroid hormones are seen more with malignant

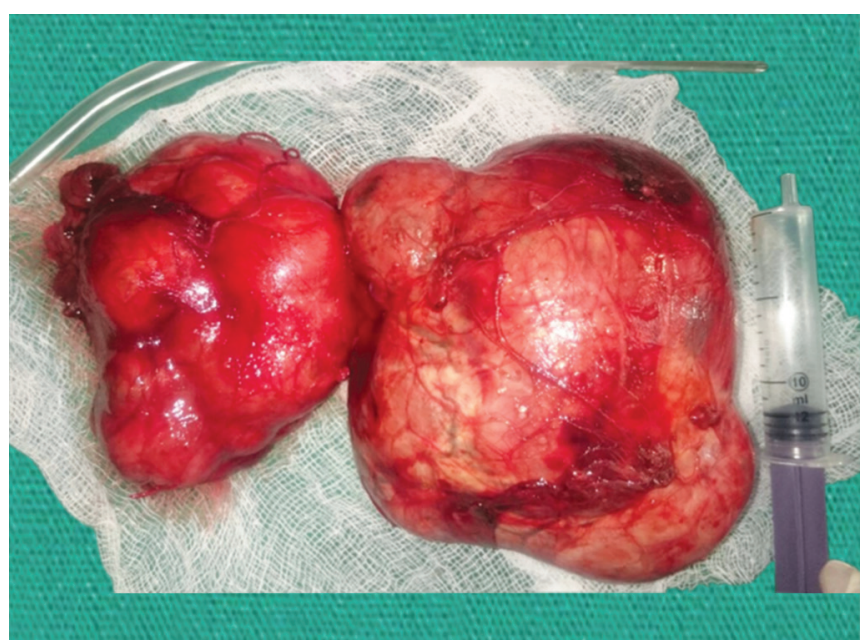

Fig. 6: Total thyroidectomy specimen with left lobe weighing $500 \mathrm{~g}$ and right lobe, $250 \mathrm{~g}$

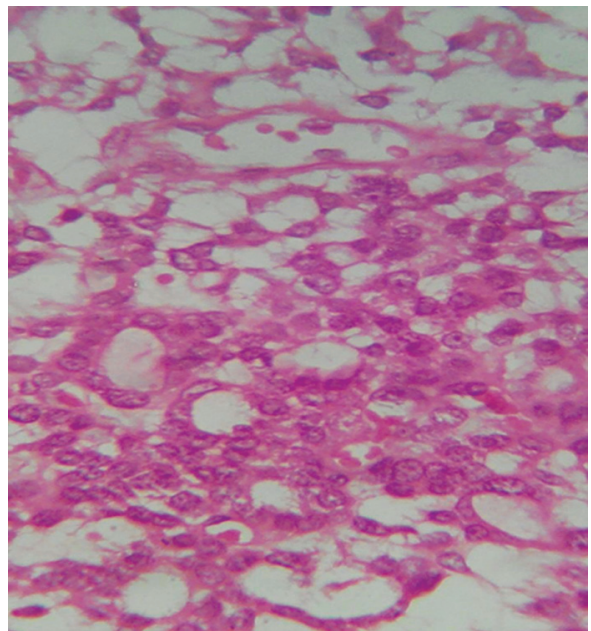

Fig 8: Histopathology demonstrating parathyroid adenoma

lesions. Intrathyroid parathyroid adenomas are extremely rare tumors. ${ }^{6}$ Preoperative localization and intraoperative guidance are needed to reduce operative failures. Preoperative SPECT in suspected cases gives accurate 3D information on ectopic adenomas. In our case report, the patient was asymptomatic with normal serum calcium levels prior to surgery. Hence parathormone levels were not measured prior to surgery.

Theodor Kocher recognized three main postoperative complications of total thyroidectomy namely myxedema, recurrent laryngeal nerve injury and tetany. ${ }^{15}$ Hypocalcemic tetany manifests as neuromuscular symptoms like circumoral paresthesia, carpopedal spasm, etc. Intracapsular dissection markedly reduces the incidence of accidental parathyroid removal. But large volume goiter, total thyroidectomy, recurrent goiter are associated with high risk of post thyroidectomy hypocalcemia. The onset of hypocalcemia occurs usually within 6 hours. Intraoperative assay of parathormone is a good but expensive tool for predicting postoperative hypocalcemia. ${ }^{16,17}$

\section{Conclusion}

This case report aims to emphasize the existence of congenital hypothyroidism and also that huge goiter when dissected meticulously 
may be removed without any major complications. We also report an incidental finding of rare intrathyroidal parathyroid adenoma.

\section{Clinical Significance}

Neonatal screening of hypothyroidism and its subsequent treatment can prevent mental retardation. We would also like to emphasize the importance of preoperative serum parathormone and calcium levels in all patients undergoing thyroidectomy to detect asymptomatic parathyroid adenomas.

\section{Ethical Approval}

All procedures performed in studies involving human participants were in accordance with the ethical standards of the institutional and/ or national research committee and with the 1964 Helsinki declaration and its later amendments or comparable ethical standards.

This article does not contain any studies with animals performed by any of the authors.

\section{Informed Consent}

Informed consent was obtained from all individual participants included in the study.

\section{References}

1. Haque $F$, Ghosh AK, Adhikari B, et al. Huge thyroid swelling - how we managed the surgical difficulties: a case report. J Evolution Med Dent Sci 2016;5(17):885-889. DOI: 10.14260/jemds/2016/204.

2. Nada A, Ahmed AM, Vilallonga R, et al. A Giant Euthyroid Endemic Multinodular Goiter with No Obstructive or Compressive Symptom. Case Rep Med 2011;2011:620480. DOI: 10.1155/2011/620480.

3. Toublanc JE. Comparison of epidemiological data on congenital hypothyroidism in Europe with those of other parts in the world. Horm Res 1992;38(5-6):230-235. DOI: 10.1159/000182549.

4. Srivastav A, Maisnam I, Dutta D, et al. Cretinism revisited. Indian J Endocrinol Metab 2012 Dec;16(Suppl 2):S336-S337. DOI: 10.4103/2230-8210.104081.
5. Sekine $\mathrm{O}$, Hozumi $\mathrm{Y}$, Takemoto $\mathrm{N}$, et al. Parathyroid adenoma without hyperparathyroidism. Jpn J Clin Oncol 2004;34(3):155-158. DOI: 10.1093/jjco/hyh028.

6. Kaushal DK, Mishra A, Mittal N, et al. Successful removal of intrathyroidal parathyroid adenoma diagnosed and accurately located preoperatively by parathyroid scintigraphy (SPECT-CT). Indian J Nucl Med 2010;25(2):62-63. DOI: 10.4103/0972-3919.72689.

7. Machado NO. Thyroidectomy for Massive Goiter Weighing more than 500 Grams. Technical Difficulties, Complications and Management. Review. Surg Sci 2011;2:278-284. DOI: 10.4236/ss.2011.25060.

8. Park SM, Chatterjee VKK. Genetics of congenital hypothyroidism. J Med Genet 2005 May;42(5):379-389. DOI: 10.1136/jmg.2004.024158.

9. Bongers-Schokking JJ, Koot HM, Wiersma D, et al. Influence of timing and dose of thyroid hormone replacement on development in infants with congenital hypothyroidism. J Pediatr 2000 Mar;136(3):292-297. DOI: $10.1067 / \mathrm{mpd} .2000 .103351$

10. Dere K, Teksoz E, Sen H, et al. Anaesthesia in a Child with Massive Thyroid Enlargement. Paediatr Anaesth 2008;18(8):797-798. DOI: 10.1111/j.1460-9592.2008.02547.x.

11. Nael K, Hur J, Bauer A, et al. Dynamic 4D MRI for characterization of parathyroid adenomas: Multiparametric analysis. AJNR Am J Neuroradiol 2015 Nov;36(11):2147-2152. DOI: 10.3174/ajnr.A4425.

12. Day TA, Chu A, Hoang KG. Multinodular Goiter. Otolaryngol Clin North Am 2003;36:35-54.

13. El Bashier EM, Widtalla ABH, Ahmed ME. Tracheostomy with Thyroidectomy: Indications, Management and Outcome: A Prospective Study. Int J Surg 2008;6(2):147-150. DOI: 10.1016/ j.ijsu.2008.01.010.

14. Tahim AS, Saunders J, Sinha P. A Parathyroid Adenoma: Benign Disease Presenting with Hyperparathyroid Crisis. Case Rep Med 2010;2010:596185. DOI: 10.1155/2010/596185.

15. Nair CG, Babu MJC, Menon R, et al. Hypocalcaemia following total thyroidectomy: An analysis of 806 patients. Indian J Endocr Metab 2013;17(2):298-303. DOI: 10.4103/2230-8210.109718.

16. Liu Q, Djuricin G, Prinz RA. Total Thyroidectomy for Benign Thyroid Disease. Surgery 1998;123(1):2-7.

17. Thomusch O, Machens A, Sekulla C, et al. The impact of surgical technique on postoperative hypoparathyroidism in bilateral thyroid surgery: A multivariate analysis of 5846 consecutive patients. Surgery 2003;133:180-185. DOI: 10.1067/msy.2003.61. 